\title{
Continuous wave Nd:YAG laser photocoagulation in proliferative diabetic retinopathy
}

U Menchini, P Lanzetta, F Soldano, E Ferrari, G Virgili

\begin{abstract}
Aims-The therapeutic efficacy of the continuous wave (CW) Nd:YAG laser (working in the free running mode) was investigated in proliferative diabetic retinopathy (PDR) comparing it with a conventional laser source such as the krypton laser.
\end{abstract}

Methods-Twenty four eyes of 12 patients affected with bilateral PDR were included and divided in two groups. The right eyes were treated with a red krypton laser and the left eyes with a CW Nd:YAG laser.

Results-Three months after krypton photocoagulation 10 eyes showed a reduction or a complete regression of PDR and two eyes were unchanged. In the $C W$ Nd:YAG laser group no eyes showed any regression of new vessels, in seven eyes the angiographic features were unchanged, and in five eyes they worsened. All the eyes of the second group underwent retreatment with the krypton laser after 3 or 6 months. After a mean follow up of 13 months all eyes in the krypton group showed a reduction or complete regression of PDR; in the retreated group 10 eyes improved and two were unchanged, Conclusions-The statistical analysis showed a highly significant difference $(p=0.001)$ between krypton and $C W$ Nd:YAG laser which indicated the lack of efficacy of the latter in the treatment of PDR. In the krypton laser group no significant difference $(p=0.05)$ after the retreatment was found confirming the efficacy of this treatment.

(Br F Ophthalmol 1995; 79: 642-645)

The efficacy of panretinal photocoagulation (PRP) in reducing the risk of severe visual loss and neovascular glaucoma in proliferative diabetic retinopathy (PDR) has been demonstrated. 12

The laser sources used nowadays to perform PRP are the continuous wave (CW) gas ion lasers such as argon blue-green (488 nm-514 $\mathrm{nm}$ ), krypton (647 $\mathrm{nm}$ ), and the solid state diode laser emitting at 805 and $810 \mathrm{~nm}$. $^{3-10}$

Recently the CW Nd:YAG laser (working in the free running mode) emitting at $1064 \mathrm{~nm}$ was introduced as a universal laser apparatus allowing the ophthalmologist to make maximum use of the increasingly wide spectrum of laser treatment options. ${ }^{1112}$

The efficacy of this infrared wavelength in retinochoroidal photocoagulation has been previously postulated ${ }^{11} 12$ and the aim of this study was to verify its role in the treatment of PDR.

\section{Subjects and methods}

Twenty four eyes of 12 patients affected with bilateral PDR were included. The mean age was 60.3 (range 41-76; SD 9.59) years; there were seven women and five men. All the patients gave their consent to the trials.

Inclusion criteria were considered to be PDR with disc new vessels or neovascularisation along the vascular arcades diagnosed by ophthalmoscopic examination and fluorescein angiogram (Fig 1A).

Patients who underwent previous laser treatment or who had vitreous haemorrhage or tractional retinal detachment were excluded from the study.

The pretreatment evaluation consisted of a visual acuity test (VA) and tonometry in each eye, colour retinography, and fluorescein angiogram.

The study was conducted according to the self matching case-control technique in order to compare the efficacy of the CW Nd:YAG and krypton laser treatment.

The eyes to be treated were divided in two groups. The right eyes were treated with a krypton laser (Ophthalas, Alcon Biophysic) (group K) and the left eyes were treated with a CW Nd:YAG laser (Microruptor III, Lasag) (group Y).

The aim of the treatment was to perform a PRP producing the ablation of the ischaemic peripheral and mid-peripheral retina, avoiding the retinal areas inside the temporal vascular arcades.

In both groups we performed a transpupillary laser treatment after the induction of a complete mydriasis with topical tropicamide $0.50 \%$ and phenylephrine $10 \%$, and a topical anaesthesia with oxybuprocaine $0.4 \%$ and the use of contact lenses with methylcellulose $2 \%$.

With the krypton laser we used the Goldmann three mirrors and the Volk quadraspheric lens, both with antireflex treatment.

The first was used for the coagulation of the peripheral retina, the second for the coagulation of mid-peripheral retina to the vascular arcades. With the CW Nd:YAG laser we used the appropriate Fankhauser lens (Lasag) (CGR3), the Goldmann three mirror lens, and the Volk quadraspheric lens.

During the post-treatment evaluation the patients were examined 2,6 , and 12 weeks after the treatment. Retinal angiograms were performed 3, 6, 9, and 12 months after the photocoagulation.

The degree and the extent of regression of PDR in the treated eyes were assessed by fluorescein angiogram and colour retinography. The response to treatment was evaluated by a three point scale according to the following 


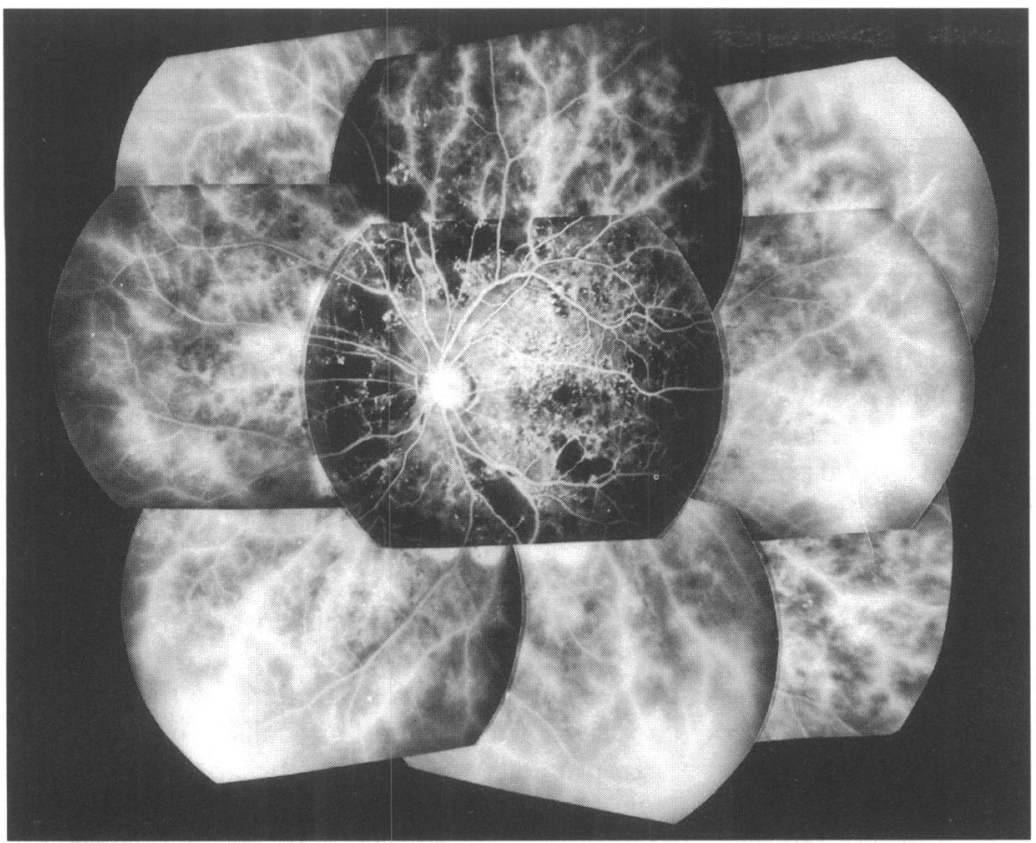

Fig 1A

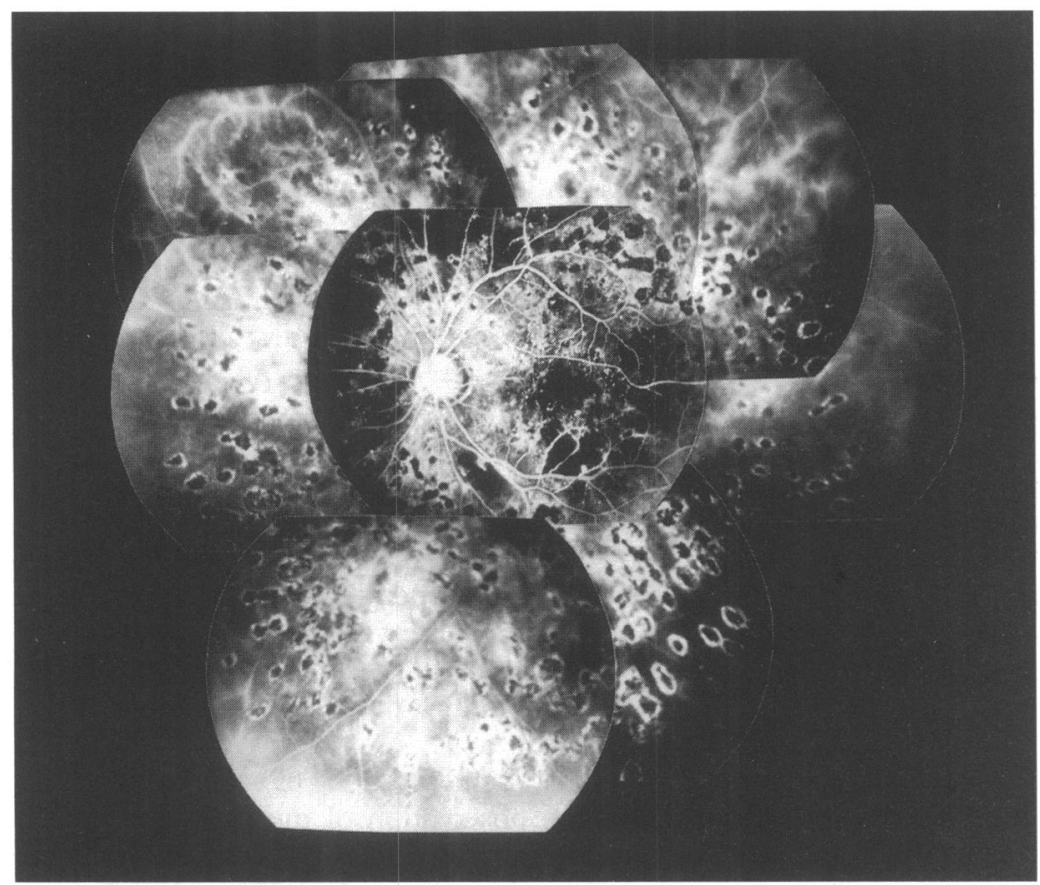

Fig 1B

Figure 1 Fluorescein angiogram before $(A)$ and 3 months after continuous wave Nd:YAG laser retinal photocoagulation (B). The excessive rarefaction of the spots did not allow the ablation of the ischaemic retina. After these results the patient underwent a laser treatment with krypton laser.

\section{TREATMENT VARIABLES}

In group $\mathrm{K}$ the mean power was 500 (range $300-700) \mathrm{mW}$ with a spot diameter of $700 \mu \mathrm{m}$ with the Goldmann lens and $300 \mu \mathrm{m}$ with the Volk lens.

The exposure was set in continuous mode and was determined by the physician on the basis of the whitening effect of laser coagulation. The mean number of spots was 206 for each session.

The criteria for the treatment with the $\mathrm{CW}$ Nd:YAG laser were: spot diameter of $400 \mu \mathrm{m}$ with the Fankhauser lens and 200-250 $\mu \mathrm{m}$ with the Volk. We stopped using the Goldmann lens because of the difficulty in obtaining visible retinal photocoagulation with powers below $6 \mathrm{~W}$. The mean power was $4.5 \mathrm{~W}$ (range $2-5 \cdot 7 \mathrm{~W}$ ).

We increased the power by about $10 \%$ with the quadraspheric lens. The exposure time, which we conventionally fixed at 2 seconds, was determined by the physician after a mild whitening of the retina as an effect of the laser.

\section{DIFFICULTIES DURING THE TREATMENT}

We did not have any particular difficulty in the use of the krypton laser. However, during treatment with the CW Nd:YAG laser we had the following problems: localisation of the laser spots and photocoagulation of the peripheral retina.

The localisation was often difficult because the operator could not determine precisely the edges of the spots. This is only possible with the lasers emitting in the visible or near infrared wavelengths (514-805 $\mathrm{nm})$. As a result, it was impossible to perform with certainty a PRP sufficient to reduce or eliminate the neovascular factor deriving from the ischaemic retinal areas.

A further problem was the impossibility of photocoagulating the mid-periphery with the Fankhauser lens, while it was also very difficult with the quadraspheric lens.

In all the eyes treated with the CW Nd:YAG laser, in fact, we obtained an insufficient ablation of the non-perfused areas. This was testified both by the excessive rarefaction of the spots along the vascular arcades and in the mid-periphery, and the unsuccessful treatment of the lasting ischaemic retinal areas (Fig 1B).

criteria: $1=$ improved: regression of the number and extension of proliferative phenomena; 2 =unchanged: no regression of the number and extension of proliferative phenomena; $3=$ worsened: increase of the number and extension of proliferative phenomena. The presence of any side effect was also recorded.

The comparison between the treatments was carried out by means of the sign test measuring the direction of the distances among the scores for every paired couple.

\section{Results}

The mean follow up time was 13 months (range 6-18 months).

\section{ANATOMICAL OUTCOME}

Three months after the laser treatment in group $\mathrm{K} 10$ eyes $(83.3 \%)$ showed a reduction or a complete regression of PDR and two eyes $(16.6 \%)$ were unchanged.

In group $\mathrm{Y}$ no patient showed any regression of the neovascularisation; in seven eyes $(58.3 \%)$ the angiographic features were unchanged and in five eyes $(41.7 \%)$ they worsened (Table 1). In all the cases, owing to the impossibility of photocoagulating the peripheral retina with the CW Nd:YAG laser, krypton laser after 3 or 6 months (group $Y+K$ ).

After a mean follow up of 13 months all the the treatment had to be completed with the eyes in group $\mathrm{Y}$ had been retreated with 
Table 1 Anatomical outcome in 12 pairs of eyes (\%) 3 months after continuous wave (CW) Nd:YAG and krypton laser treatment (evaluated with fundus photograph and fluorescein angiogram)

\begin{tabular}{lcll}
\hline & Improved & Unchanged & Worsened \\
\hline CW Nd:YAG laser $(\mathrm{n}=12)$ & $0(0 \cdot 0)$ & $7(58 \cdot 3)$ & $5(41 \cdot 7)$ \\
Krypton laser $(\mathrm{n}=12)$ & $10(83 \cdot 3)$ & $2(16 \cdot 6)$ & $0(0 \cdot 0)$
\end{tabular}

krypton laser and we recorded the following data: in group $\mathrm{K} 12$ eyes $(100.0 \%)$ showed a reduction or a complete regression of PDR; in group $\mathrm{Y}+\mathrm{K}$ a regression of neovascularisation was found in 10 eyes $(83.3 \%)$, while in two eyes $(16 \cdot 7 \%)$ the angiogram features were unchanged (Table 2).

It is remarkable how the eyes that improved in group $\mathrm{Y}(10,83.3 \%)$ underwent a krypton laser treatment 3 or 6 months after the first photocoagulation with the $\mathrm{CW} N \mathrm{Nd}$ :YAG laser since no regression of the PDR was detectable in the angiogram.

The statistical analysis was conducted in two parts: a first comparison 3 months after the treatment in order to assess the responses to the different techniques; then, after the treatment was completed in group $\mathrm{Y}$ with the krypton laser, a second comparison was done taking into account the whole follow up from the beginning of the trial to the final assessment of the retinal response to krypton or CW Nd:YAG plus krypton laser treatment.

In the results obtained for the 12 pairs of eyes (Tables 3a, 3b) (the last column indicates the signs of the differences within each pair) we noted in the first comparison a highly significant difference $(p=0.001)$, confirming the inefficacy of CW Nd:YAG laser treatment in PDR (Table $3 \mathrm{a}$ ); in the second comparison no significant difference $(p=0.05)$ between the groups ( $\mathrm{K}$ and $\mathrm{Y}+\mathrm{K}$ ) was found confirming the efficacy of the krypton laser photocoagulation.

\section{CLINICAL SIDE EFFECTS}

In group $\mathrm{K}$ we had the following complications: in two eyes a serous choroidal detachment was observed; it cleared up spontaneously in a period of 7 days at most.

All the patients reported an internal ophthalmoplegia, illustrated by the accommodation troubles and the pupillary hyporeflexia.

All the patients complained of pain during photocoagulation corresponding with the intrachoroidal path of the long ciliary nerves. We did not notice the appearance or worsening of lens opacities.

In group $\mathrm{Y}$ six eyes showed a serous choroidal detachment that spontaneously cleared up in about 7 days.

Table 2 Anatomical outcome in 12 pairs of eyes (\%) after continuous wave (CW) Nd:YAG plus krypton and krypton laser treatment (evaluated with fundus photograph and fluorescein angiogram). Mean follow up 13 (range 6-18) months

\begin{tabular}{|c|c|c|c|}
\hline & Improved & Unchanged & Worsened \\
\hline $\begin{array}{l}\text { CW Nd:YAG plus krypton } \\
\text { laser }(n=12) \\
\text { Krypton laser }(n=12)\end{array}$ & $\begin{array}{l}10(83 \cdot 3) \\
12(100)\end{array}$ & $\begin{array}{l}2(16 \cdot 7) \\
0(0.0)\end{array}$ & $\begin{array}{l}0(0 \cdot 0) \\
0(0.0)\end{array}$ \\
\hline
\end{tabular}

Table $3 a$ Retinal response to laser treatment in the groups 3 months after photocoagulation ( $1=$ improved; $2=$ unchanged; $3=$ worsened

\begin{tabular}{llll}
\hline Subjects & Group $K$ & Group $Y$ & Sign \\
\hline 1 & 2 & 2 & $=$ \\
2 & 1 & 2 & + \\
3 & 1 & 2 & + \\
4 & 2 & 3 & + \\
5 & 1 & 3 & + \\
6 & 1 & 3 & + \\
7 & 1 & 3 & + \\
8 & 1 & 3 & + \\
9 & 1 & 2 & + \\
10 & 1 & 2 & + \\
11 & 1 & 2 & + \\
12 & 1 & & \\
\hline
\end{tabular}

$\mathrm{p}<0.001$.

With the quadraspheric lens, six eyes had an accidental photocoagulation of the pupil border with a transfer of energy to the anterior capsule of the lens and a localised opacity.

In one eye we reported the appearance of a lens opacity without any accidental photocoagulation of the iris. In all the cases an internal ophthalmoplegia was noted. The ophthalmoplegia and pain occurred in all the patients even if the photocoagulation was performed near the posterior pole, where the ciliary nerves are still deep in the choroid.

The chorioretinal atrophy obtained with the CW Nd:YAG laser is very similar to that described for the diode laser. Its dimensions are larger than the nominal spot, probably because, owing to the infrared wavelength, there is a greater choroidal involvement and thus more extended atrophic phenomena.

\section{Discussion}

We believe that the failure of the CW Nd:YAG laser treatment depended on two main factors: the first connected with the characteristics of the slit-lamp of the photocoagulator and the second resulting from the characteristics of the wavelength.

(1) The configuration of Microruptor III makes the observation of the peripheral retina extremely difficult because coaxial illumination is not possible. The possibilities of observing and performing a correct photocoagulation are thus limited.

The incomplete photocoagulation of the peripheral retina - that is, the non-perfused areas, is certainly the main reason for the

Table $3 b$ Retinal response to laser treatment in the groups after a mean follow up of 13 (range 6-18) months (1=improved; $2=$ unchanged; $3=$ worsened

\begin{tabular}{llll}
\hline Subjects & Group $K$ & Group $Y$ & Sign \\
\hline 1 & 1 & 1 & $=$ \\
2 & 1 & 1 & $=$ \\
3 & 1 & 1 & $=$ \\
4 & 1 & 1 & $=$ \\
5 & 1 & 2 & $=$ \\
6 & 1 & 1 & $=$ \\
7 & 1 & 2 & $=$ \\
8 & 1 & 1 & $=$ \\
9 & 1 & 1 & $=$ \\
10 & 1 & 1 & \\
11 & 1 & 1 & \\
12 & 1 & & \\
\hline $\mathrm{p}=0.05$. & & &
\end{tabular}


unsuccessful regression of neovascular proliferations in the patients that we treated.

The accidental photocoagulation of the iris may be attributed to the difficulties during the treatment of the periphery as well.

(2) The wavelength of the CW Nd:YAG laser is minimally absorbed by the retinal pigment epithelium and the choroidal pigment $(2-10 \%)$, while the percentage of absorbance is much higher for the krypton laser $(60-70 \%) .{ }^{13}$ This results in a visible photocoagulation of the retina but only at high levels of energy which determine photocoagulation of the inner retinal layers.

Fankhauser et al have reported on clinical use of the CW Nd:YAG laser in background diabetic retinopathy. The irradiation was performed using the three mirror Goldmann contact lens with $20 \mathrm{~ms}$ free running Nd:YAG laser pulse of 600 to $1000 \mathrm{~mJ} .^{12}$ This is a high power $(30-50 \mathrm{~W})$ when compared with the usual effective power for retinal whitening with argon and krypton laser.

In order to have any visible effect, high powers must be applied with the Nd:YAG laser, with a consequent double disadvantage: a deeper penetration in the choroidal layers with larger atrophy and a greater dispersion of the energy through the dioptric media. They absorb about $30 \%$ of the radiation, with a consequent danger for cataract or increased vitreoretinal tractions. ${ }^{14}$ The mean power that we used was $4.5 \mathrm{~W}$, so that about $1.3 \mathrm{~W}$ was absorbed by the cornea, the lens, and the vitreous.

This fact may partly explain the occurrence of cataract after the laser treatment without iris involvement. The danger of cataract is much greater if an accidental photocoagulation of the pupillary border occurs; in this case almost the whole of the energy is absorbed by the target with consequent damage to the iris and the lens. Also the absorbance by the cornea and the vitreous body should be evaluated. In our study we did not notice any specific damage but this has to be considered.

Particularly when using the quadraspheric contact lens, the plane of highest power density is moved from the fundus towards the cornea when the spot size is set to larger than $50 \mu \mathrm{m}$. Owing to the optical properties of the lens the beam diameter at the cornea is reduced by a factor of 2 which increases the power density at the cornea by a factor of 4 . Still, the important factor is the absorption property of the cornea and other optic media related to the wavelength.

In the case of vitreal fibrosis the increase of tractional forces on the retina might cause a secondary retinal detachment. On the other hand the aim of the treatment is to destroy the non-perfused areas. In order to obtain this effective burn, the retina must be involved in the photocoagulation, while the destruction of the underlying choroid does not seem to be necessary.

The complete photocoagulation of the ischaemic tissue depends on a visible track that is, whitening of the retina, lasting for some minutes as described for the diode laser. ${ }^{15}$

This well marked track is not possible with the $\mathrm{CW} N d$ :YAG laser without applying very high powers with the ensuring risks that we have described.

1 Diabetic Retinopathy Group. Photocoagulation of proliferative diabetic retinopathy study findings. Ophthalmology tive diabetic retin

2 Diabetic Retinopathy Study Research Group Photocoagulation treatment of proliferative diabetic retinopathy: clinical application of diabetic retinopathy study (DRS) findings. DRS report no 8. Ophthalmology 1981; 88: 583-660.

3 Blankenship GW. Red krypton and blue-green argon panretinal laser photocoagulation for proliferative diabetic retinopathy: a laboratory and clinical comparison. Tran Am Ophthalmol Soc 1986; 84: 967.

4 Brancato R, Bandello F, Trabucchi G, Leoni G, Lattanzio $R$. Argon and diode laser photocoagulation in proliferative diabetic retinopathy: a preliminary report. Lasers and Light in Ophthalmology 1990; 3: 233-7.

5 Brancato R, Pratesi R. Applications of diode laser in ophthalmology. Lasers and Light in Ophthalmology 1987; 1: 119-24.

6 Brancato R, Pratesi R, Leoni G, Vanni U. Histopathology of diode and argon laser in rabbit chorioretina: a comparative study. Invest Ophthalmol Vis Sci 1989; 7: 1504-10.

7 Brancato R, Menchini U. Microchirurgia laser in oftalmologia. Milan: Ghedini editore, 1989.

8 L'Esperance FA. Clinical photocoagulation with the krypton laser. Arch Ophthalmol 1972; 87: 693.

9 Puliafito CA. Semiconductor diode laser photocoagulation in retinal vascular diseases. Laser in Surgery and Medicine 19902 (suppl): 67.

10 Singerman LJ, Ferris III FL, Mowery RP, Brucker AJ, Lerner BC, Mincey GJ. Krypton laser for proliferative diabetic retinopathy. $\mathcal{F}$ Diab Complic 1988; 2: 189-96.

11 Fankhauser F, Kwasniewska S. A new universal laser apparatus for intra and extraocular irradiation tasks in the eye Ophthalmic Surg 1990; 4: 258-62.

12 Fankhauser F, Van Der Zypen E, Kwasniewska S, Loertscher $H$. The effect of thermal mode Nd:YAG laser radiation on vessels and ocular tissues. Ophthalmology 1985; 3: 419-26.

13 Gabel VP, Birngruber R, Hillenkamp F. Visible and near infrared light absorption in pigment epithelium and choroid. In: Shimizu K, ed. International Congress Series no 450, XXIII Concilium Ophthalmol Kyoto. Amsterdam, 450, XXIII Concilium Ophthalmol Kyoto. Amsterdar
Oxford: Excerpta Medica, Elsevier, 1978: 658-62.

14 Wiesinger H, Schmidt TJ, Williams RC, Tiller CO, Ruffin RS, Guerry D III, et al. The transmission of light through the rabbit eye. Am $\mathcal{f}$ Ophthalmol 1956; 42: 907-10

15 McHugh JDA, Marshall J, Fytche TJ, Hamilton AM, Raven A. Initial clinical experience using diode laser in the treatment of retinal vascular disease. Eye 1989; 3: 516-27. 